\title{
Numerical Investigation of Spectral Evolution of Wind Waves. Part II: Dissipation Term and Evolution Tests
}

\author{
ALEXANDER V. BABANIN \\ Faculty of Engineering and Industrial Science, Swinburne University of Technology, Melbourne, Victoria, Australia
}

\author{
KAKHA N. TSAGARELI
}

School of Civil, Environmental and Mining Engineering, The University of Adelaide, Adelaide, South Australia, Australia

\author{
I. R. YOUNG
}

Swinburne University of Technology, Melbourne, Victoria, Australia

\section{DAVID J. WALKER}

School of Civil, Environmental and Mining Engineering, The University of Adelaide, Adelaide, South Australia, Australia

(Manuscript received 29 September 2009, in final form 24 November 2009)

\begin{abstract}
Numerical simulations of the wind-wave spectrum evolution are conducted by means of new observationbased wind-input and wave dissipation functions obtained in the Lake George field experiment. This experiment allowed simultaneous measurements of the source functions in a broad range of conditions, including extreme wind-wave circumstances. Results of the experiment revealed new physical mechanisms in the processes of spectral input/dissipation of wave energy, which are presently not accounted for in wave forecast models. These features had been parameterized as source terms in a form suitable for spectral wave models; in the present study, they were tested, calibrated, and validated on the basis of such a model.

Physical constraints were imposed on the source functions in terms of the known experimental dependences for the total wind-wave momentum flux and for the ratio between the total input and total dissipation. Enforcing the constraints in the course of wave-spectrum evolution allowed calibration of the free experimental parameters of the new input (Part I of the study) and dissipation functions; the latter is the topic of the present paper. The approach allows separate calibration of the source functions before they are employed in the evolution tests. The evolution simulations were conducted by means of the one-dimensional research WAVETIME model with an exact solution for the nonlinear term. The resulting time-limited evolution of integral, spectral, and directional wave properties, based on implementation of the new physically justified source/sink terms and constraints, is then analyzed. Good agreement of the simulated evolution with known experimental dependences is demonstrated.
\end{abstract}

\section{Introduction}

The dissipation term $S_{\mathrm{ds}}$ is one of the three major source functions of the radiative transfer equation (RTE), which in deep water can be formulated as

$$
\frac{d F}{d t}=S_{\mathrm{in}}+S_{\mathrm{nl}}+S_{\mathrm{ds}}+\cdots
$$

Corresponding author address: Alexander V. Babanin, P.O. Box 218, Hawthorn, VIC 3122, Australia.

E-mail: ababanin@swin.edu.au
One of the most common applications of this equation is its use by spectral wave forecast models, which are intended to predict the wave spectrum $F$. The two other source terms of wind input $S_{\text {in }}$ and resonant nonlinear four-wave interactions $S_{\mathrm{nl}}$ are also explicitly mentioned here; however, in a general case, many more source/sink terms are possible. All the source terms as well as the spectrum itself are functions of wavenumber $\boldsymbol{k}$, frequency $\omega$, time $t$, and spatial coordinate $\boldsymbol{x}$.

When wave breaking is present, the dominant part of $S_{\mathrm{ds}}$ is attributed to energy losses resulting from such breaking. The breaking has been routinely regarded as 
a poorly understood phenomenon; therefore, formulations of the dissipation term have always been loosely based on physics and served as a residual tuning knob (e.g., Cavaleri et al. 2007; Babanin and van der Westhuysen 2008). In a growing number of recent publications, however, it was shown that such an approach is no longer satisfactory (e.g., Babanin et al. 2007b,c; Babanin and van der Westhuysen 2008; Ardhuin et al. 2009, manuscript submitted to J. Phys. Oceanogr., hereafter AR; Babanin 2009). Experimental advances over the past decade have revealed new physical features of the wave breaking process and spectral dissipation.

The first such feature was the threshold behavior of wave breaking observed by Banner et al. (2000) and Babanin et al. (2001). Based on observations of wave breaking in a very broad range of circumstances and wave scales, from a small lake to the Southern Ocean, from deep water to finite depths, and from mature waves to very strongly wind-forced conditions, it was shown that waves will not break unless some average background steepness exceeds a threshold value. Once this value is overcome, the breaking frequency/probability will depend on the excess of the mean steepness above the threshold.

To convert the threshold estimates into formulations suitable for spectral models, Babanin and Young (2005), Young and Babanin (2006), and Babanin et al. (2007c) suggested a quantitative analog of the spectral threshold value and a new dissipative term that accommodates the threshold behavior. The new term was then tested in a research spectral model (Babanin et al. 2007b; Tsagareli 2008) alongside a new wind-input term (Donelan et al. 2006) and a full solution to the nonlinear integral (van Vledder 2002, 2006). Van der Westhuysen et al. (2007) using the Simulating Waves Nearshore (SWAN) model and AR in WAVEWATCH-III implemented alternative formulations, which incorporated the threshold behavior, and conducted a series of tests and hindcasts. Overall, however, although such findings have obvious implications for the dissipation function $S_{\mathrm{ds}}$, implementation of the threshold in operational wave models has not occurred. As a result, many present-day models treat wave evolution in certain circumstances in quite unrealistic ways. For instance, when the waves are known not to be breaking, the whitecapping dissipation in such models is still active (for details and comments, see, e.g., Babanin 2009).

Another important feature of spectral dissipation is the cumulative dissipative effect. This effect is due to breaking and/or dissipation of short waves being influenced or even directly induced by longer waves. Although the induced breaking/dissipation has been observed for a substantial period of time (e.g., Banner et al. 1989; Melville et al. 2002; Manasseh et al. 2006), the effect has been overlooked by the wave modeling community and until very recently not accounted for in formulations of $S_{\mathrm{ds}}$.

The significance of cumulative dissipation in the spectral environment is most prominent. Although the majority of present-day spectral models explicitly link the dissipation at a specific scale $S_{\mathrm{ds}}(\omega, \mathbf{k})$ with the spectrum $F(\omega, \mathbf{k})$ at that scale, this connection has actually only been observed for a narrow frequency band around the spectral peak (see Babanin and Young 2005; Manasseh et al. 2006; Young and Babanin 2006; Babanin et al. 2007b,c; AR; Babanin 2009). Donelan (2001) was the first to point this out and to suggest an $S_{\mathrm{ds}}$ formulation that accounts for the cumulative effect resulting from breaking caused by compression of short waves riding the longer waves.

A different kind of cumulative dissipation was observed by Babanin and Young (2005) and Young and Babanin (2006). They demonstrated that breaking of dominant waves brings about a broadband impact. As a result of such breaking at the spectral peak, the spectral energy is dissipated across the entire frequency range (at least up to $10 \mathrm{~Hz}$ in their measurements where the peak frequency was $f_{p} \sim 0.4 \mathrm{~Hz}$ ). A new form of the dissipation function $S_{\mathrm{ds}}$ was proposed that accounts for the "accumulation" of dissipation and quantifies it within the limits of the measurement. As mentioned earlier, this formulation also accommodates the threshold behavior, which was also quantified (for further developments, see also Babanin et al. 2007c; Babanin 2009).

As with the threshold behavior, however, the cumulative term of the dissipation function is yet to make its way into operational modeling. In this regard, it is only a recent attempt by AR that can be mentioned where an alternative to Donelan (2001) and to Babanin and Young (2005) formulation of the cumulative term was suggested and applied in the WAVEWATCH-III model for hindcasting a broad range of wind-wave situations, with a noticeable improvement of the wave predictions in some circumstances. It should be mentioned that, in the standard version of WAVEWATCH-III, two-phase behavior of the dissipation term is already accommodated (i.e., formulations for the dissipation function at the spectral peak and at the tail are different), but this is not the cumulative term, and the assumed physics of Tolman and Chalikov (1996) is different to that suggested by Donelan (2001) and that observed in the experiments by Babanin and Young (2005), Manasseh et al. (2006), and Young and Babanin (2006).

Among further new developments with respect to physics-based, rather than fitting- and tuning-based dissipation functions, the ongoing effort by Filipot et al. (2008, 2010) should be noted. In this approach, the 
dissipation term is obtained as a product of wave-breaking probability and severity. Filipot et al. use a combination of analytical and experimental means to quantify the spectral distribution of the probability and severity, and the resulting dissipation exhibits both the threshold and cumulative behavior [see also the earlier experimental attempts by Manasseh et al. (2006) and the detailed discussion of such approach in Babanin (2009)].

Other newly observed features of the dissipation function should also be mentioned. These include quasisingular behavior of dissipation in the middle wavelength range (Hwang and Wang 2004), alteration of the wave breaking/dissipation at strong wind forcing (Babanin and Young 2005; Babanin 2009), and bimodal directional distribution of the dissipation function (Young and Babanin 2006). The significance of these additional features is still to be investigated [see the attempts to introduce directionality into $S_{\mathrm{ds}}$ by Babanin et al. (2007b), Tsagareli (2008), and AR and the discussion in Babanin (2009)].

It should be pointed out that introducing new source terms or updating existing terms, based on advanced understanding of physics of waves and air-sea interactions, does not necessarily immediately lead to improvements of the hindcast/forecast. Models have been fine-tuned over many years, and their forecast in standard situations is already quite good. It is nonstandard or complex situations that are likely to benefit from improved physics. Ardhuin et al. (2007) showed one such situation: wave growth in the presence of swell and at slanting fetches. The most obvious and important "nonstandard" situation that could potentially benefit from enhanced physics are extreme windwave conditions. Other examples, where standard models are known to fail include complicated seas such as those when two opposing wave systems intersect as during the infamous Sydney to Hobart yacht race (Greenslade 2001) or in the course of Typhoon Krosa approaching Taiwan (Liu et al. 2007). In such situations, both the dissipation and wind-input functions would behave in ways not accounted for by the "tuning" approaches [e.g., among most recent publications on wind input in such circumstances, Donelan et al. (2006), Babanin et al. (2007a), and Stiassnie et al. (2007)].

Incorporation of the new dissipation features, however, is more complex than simply replacing one dissipation term with another. For example, if a cumulative integral is added to the breaking dissipation term, then the local-in-wavenumber-space balance can no longer be satisfied, and reformulations and readjustments of the wind-input function, as well as perhaps the entire model, will also be required. Therefore, the effort presented in this paper includes testing and incorporation of both the new wind-input function based on the experiments by Donelan et al. (2006) and the new dissipation function following experiments by Babanin and Young (2005) and Young and Babanin (2006). Validation and calibration of $S_{\text {in }}$ is described in Tsagareli et al. (2010, hereafter Part I), and validation and calibration of the dissipation $S_{\mathrm{ds}}$ is described here.

This paper also includes wave-evolution verification by means of a standard set of tests for integral, spectral, and directional properties of wave fields. It should be highlighted in advance that the latter has been difficult in the past (e.g., Banner and Young 1994), and it is the introduction of the directionally dependent $S_{\mathrm{ds}}$ in this study that yielded improvements in this regard. The wave growth was simulated by means of the one-dimensional research model WAVETIME developed by van Vledder (2002, 2006).

The dissipation function to be implemented here is that by Babanin and Young (2005) and Young and Babanin (2006). The general approach to testing and validating it, based on physical constraints imposed on the wind-input and whitecapping terms, is outlined in section 2 . Section 3 is dedicated to a detailed description and quantitative calibration of the dissipation term across a broad range of wind-wave conditions. In section 4, the evolution tests are conducted, and the outcomes are discussed and summarized in section 5 .

\section{The approach}

The traditional approach to testing source functions employed in the RTE (1) was developed by Komen et al. (1984) and with some variations has persisted throughout more than $30 \mathrm{yr}$. This approach involves implementing a new function in the RTE, solving this equation numerically by means of a spectral wave model, and comparing the outcomes with known experimental results. These outcomes present the time-space development of integral, spectral, and directional properties of the wave field. The evolution of such wave properties has been extensively investigated and is well understood, described, and parameterized. Therefore, this approach concentrates on reproducing the known wave-growth curves and some spectral features (e.g., Banner and Young 1994), and the ability of a model to replicate these curves serves as a validation measure for the source terms.

Such validation criterion can, however, mask potentially serious defectiveness. The wind-input term supplies a positive flux of energy/momentum to the wave field, whereas the dissipation term provides a negative flux. Together, they can balance each other and produce apparently correct growth curves while being physically inadequate individually. For instance, nothing stops $S_{\text {in }}$ 
being of a form such that its integral is many times greater than a realistic total wind-energy input, provided that $S_{\mathrm{ds}}$ has a matching integral (see, e.g., Part I).

An additional disadvantage of this traditional approach is that it is impossible to investigate and verify the source terms separately. Any change to any of the source terms requires extensive testing with a full spectral model, which is computationally very expensive, particularly as the exact nonlinear term has to be solved. In addition, alterations of one term often lead to the need for compensative tuning of other source functions.

A new approach has been developed that overcomes the previously mentioned limitations. The approach is described in detail in Tsagareli (2008) and Part I and is based on physical constraints suggested by M. Donelan (2004, WISE-2004 Meeting, personal communication).

The main constraint imposes the condition that the integral wind-momentum input $S_{m}$ must be equal to the total wave-induced stress $\tau_{w}$ (i.e., total wind stress less the viscous component),

$$
\int_{0}^{f_{\infty}} S_{m}(f) d f=\int_{0}^{f_{\infty}} \frac{k}{\omega} S_{\text {in }}(f) d f=\tau_{w} .
$$

In the present study, $f_{\infty}=10 \mathrm{~Hz}$ was chosen such that it is well within the capillary wave-frequency range. Based on this constraint, behavior of a new wind-input function can be investigated and tuned separately from any other term, because dependences of the total drag on, for example, wind speed (e.g., Garratt 1977) or wave age (e.g., Guan and Xie 2004), as well as experimental dependences for the viscous drag (e.g., Banner and Peirson 1998; Part I), are well known. The new experimental wind-input function employed in this study is that by Donelan et al. (2006) obtained in the Lake George experiment (Donelan et al. 2005; Young et al. 2005).

The second main constraint relates to the dissipation function whose integral must not exceed the total wind input,

$$
\int_{0}^{f_{\infty}} S_{\mathrm{ds}}(f) d f \leq \int_{0}^{f_{\infty}} S_{\text {in }}(f) d f .
$$

The ratio of the two integrals as a function of wavedevelopment stage is also known experimentally (e.g., Donelan 1998); therefore, the dissipation term can also be studied and tuned individually. This will be discussed in the next section. The ability of the updated source terms to reproduce the growth curves and the known behavior of the wave spectral evolution are obviously still crucial, and these checks will be conducted later.

\section{The new dissipation function}

The new dissipation function was suggested on the basis of a series of experimental studies (Babanin and Young 2005; Manasseh et al. 2006; Young and Babanin 2006). New physical features of the spectral dissipation, such as the threshold behavior and the cumulative effect at smaller scales, were verified by independent and redundant means before being incorporated in the suggested dissipation function (see Babanin et al. 2007c; Babanin 2009):

$$
\begin{gathered}
S_{\mathrm{ds}}(f)=a \underbrace{f f(f)\left(F(f)-F_{T}(f)\right)}_{T_{1}(f)} \\
+b \underbrace{b \int_{f_{p}}^{f} A(q)\left(F(q)-F_{T}(q)\right) d q}_{T_{2}(f)} \text { if } \\
\\
F(f)>F_{T}(f), \\
S_{\mathrm{ds}}(f)=0 \text { if } \quad F(f) \leq F_{T}(f), \quad \text { and } \\
T_{2}(f)=0 \text { if } \quad f<f_{p} .
\end{gathered}
$$

Here, $A(f)$ is the integral characteristic of the inverse directional spectral width (Babanin and Soloviev 1987, 1998a),

$$
A(f)^{-1}=\int_{-\pi}^{\pi} K(f, \phi) d \phi
$$

where $\varphi$ is the wave direction and $K(f, \phi)$ is the directional spectrum normalized by its value in the direction $\phi_{\max }$ where the maximum is observed,

$$
K\left(f, \phi_{\max }\right)=1 .
$$

The terms $a$ and $b$ are experimental constants, and $F_{T}(f)$ is the spectral threshold function.

The dissipation function $S_{\mathrm{ds}}$ in (4) accommodates the two-phase behavior: it is a simple function of the wave spectrum at the spectral peak (inherent breaking term $T_{1}(f)$, which also persists through the entire spectrum) and has an additional cumulative term $T_{2}(f)$ at all frequencies above the peak. The latter is an integral, which obviously grows toward higher frequencies and dominates at smaller scales: that is, $T_{2}(f) \gg T_{1}(f)$ for $f \gg f_{p}$ (Babanin and Young 2005). As discussed earlier, the induced dissipation at a particular frequency above the peak can be caused by lower-frequency breaking (i.e., Babanin and Young 2005; Manasseh et al. 2006; Young and Babanin 
2006) or/and by modulation of the short-wave steepness by underlying longer waves (Donelan 2001).

Equation (4) has a number of free parameters that require experimental measurements or numerical simulations to be defined. These include the spectral threshold $F_{T}(f)$ and coefficients $a$ and $b$. Limits for the threshold value $F_{T}(f)$ were identified in the experimental study of Babanin and Young (2005) and further investigated in Babanin et al. (2007b,c), Tsagareli (2008), and Babanin (2009).

Here, we will follow Tsagareli (2008); that is, we will first employ the saturation spectrum $\sigma(f)$ normalized by the directional spectrum parameter (5).

$$
\sigma(f)=\sigma_{\text {Phillips }}(f) A(f),
$$

where $\sigma_{\text {Phillips }}(f)$ is as introduced by Phillips (1984),

$$
\sigma_{\text {Phillips }}(f)=\frac{(2 \pi)^{4} f^{5} F(f)}{2 g^{2}} .
$$

If a universal dimensionless saturation-threshold value $\sigma_{\text {thr }}$ can be established, the dimensional threshold can then be obtained at every frequency,

$$
F_{T}(f)=\frac{2 g^{2}}{(2 \pi)^{4}} \frac{\sigma_{\mathrm{thr}}}{A(f) f^{5}} .
$$

Here, the normalization by the directional width $A^{-1}(f)$ was introduced formally, following Banner et al. (2002), who found the correction for the directional spread helpful in reconciling values of the wave-breaking threshold across different spectral bands. It is now understood that the correction is not necessary and the seeming difference in threshold values observed by Banner et al. (2002) is most likely due to the cumulative dissipation toward higher frequencies (Babanin et al. 2007a; Babanin 2009). This conclusion does not essentially affect the threshold magnitude used here because $A \approx 1$ at this threshold (Babanin 2009).

Although it was tempting to obtain wave-breaking probability across the spectrum in terms of dimensionless $\sigma(f)$ and thus to determine the threshold value $\sigma_{\mathrm{thr}}$ directly, such a solution did not prove feasible. First, the saturation spectrum is the fifth moment of the spectrum; therefore, if derived from measured spectra, it is very noisy unless the spectra are in deep water and windwave conditions are very pure (Babanin and Young 2005). Second, the cumulative effect disconnects the saturation $\sigma(f)$ from the breaking occurrence rate $b_{T}(f)$ at frequencies $f$ other than the spectral peak (Babanin et al. 2007c), and this produces misleading conclusions

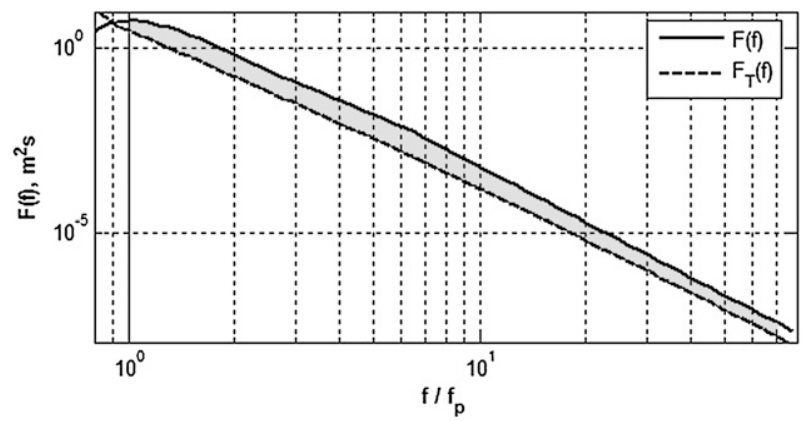

FIG. 1. Illustration of the threshold spectrum $F_{T}(f)$.

about the magnitude of $\sigma_{\mathrm{thr}}$, as mentioned earlier. This is discussed in detail by Babanin (2009). Therefore, indirect approaches to identify the $\sigma_{\text {thr }}$ limit were undertaken. In an extensive set of Lake George wavebreaking measurements across different spectral bands published by Babanin and Young (2005), it was shown that the lowest value of saturation for which the breaking was still detected was $\sqrt{\sigma(f)}=0.0255$. Babanin et al. (2007c) and Babanin (2009) argued that the bulk of Lake George data corresponds to values of the dimensionless threshold in the range of

$$
\sqrt{\sigma(f)}>0.035
$$

with only a couple of outliers falling below this value.

Babanin et al. (2007b) and Tsagareli (2008) conducted numerical simulations using the research one-dimensional model WAVETIME (see also section 4) to test the threshold (10). Simulations were run for a broad range of wind speeds of $U_{10}=7-20 \mathrm{~m} \mathrm{~s}^{-1}$, and an additional restriction was imposed that at full development (PiersonMoskowitz stage) marginal or no breaking can occur at the spectral peak. This was found to be the case if the threshold chosen is

$$
\sqrt{\sigma_{\mathrm{thr}}(f)}=\mathrm{const}=0.035 \text {. }
$$

Thresholds (10) and (11) will thus be used to find dimensional spectral threshold value $F_{T}(f)$ at each frequency according to (9).

The implication of this threshold for the wavebreaking dissipation function is illustrated in Fig. 1. Here, the threshold spectrum $F_{T}(f)$ [Eq. (9); dashed line] and a typical fully developed spectrum $F(f)$ (solid line) are shown. The dissipation is related to the excess $\Delta F(f)=F(f)-F_{T}(f)$, identified by the shaded area, rather than to the spectrum $F(f)$, and below the spectrum peak should become zero. At the spectral peak of the fully developed spectrum shown, however, the 
dissipation is small but not zero, whereas experimental data point to zero-breaking rates in such circumstances (e.g., Banner et al. 2000). Because the threshold (11) is an experimental characteristic and cannot be tuned (raised), this means that other less certain parameters of the spectral dissipation shape (4) need to be calibrated.

\section{Calibration of the dissipation term}

For the calibration tests, a special Joint North Sea Wave Project (JONSWAP)-like spectral parameterization form was chosen, which was adapted to have both subintervals of $f^{-4}$ and $f^{-5}$ behavior in the equilibrium interval,

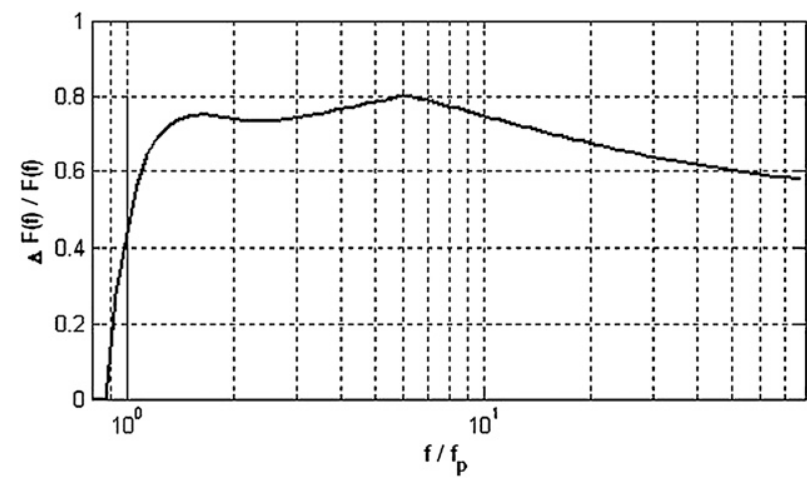

FIG. 2. Ratio of $\Delta F(f)=F(f)-F_{T}(f)$ for the fully developed Combi spectrum [Eq. (12)] vs relative frequency $f / f_{p}$. The computations were performed for fully developed conditions at a wind speed $U_{10}=10 \mathrm{~m} \mathrm{~s}^{-1}$.

$$
\begin{cases}F(f, \theta)=\alpha \frac{g^{2}}{(2 \pi)^{4}} f_{p}^{-1} f^{-4} \exp \left[-\left(\frac{f}{f_{p}}\right)^{-4}\right] \gamma_{D}^{\exp \left[-\left(f-f_{P}\right)^{2} / 2 \sigma^{2} f_{P}^{2}\right]} & f \leq f_{t} \\ F(f, \theta)=\alpha \frac{g^{2}}{(2 \pi)^{4}} f_{t} f_{p}^{-1} f^{-5} \exp \left[-\left(\frac{f}{f_{p}}\right)^{-4}\right] \gamma_{D}^{\exp \left[-\left(f-f_{P}\right)^{2} / 2 \sigma^{2} f_{P}^{2}\right]} & f>f_{t}\end{cases}
$$

where $f_{t}$ is the transition frequency and the tail level $\alpha$, enhancement parameter $\gamma_{D}$, and peak width $\sigma$ are standard JONSWAP spectrum parameters (Hasselmann et al. 1973). Similar spectral shapes, which can accommodate either type of the spectral tail, had been suggested before (e.g., Young and Verhagen 1996); here, we unite them into a single parameterization that exhibits an $f^{-4}$ dependence closer to the peak and an $f^{-5}$ dependence at higher frequencies. Following Tsagareli (2008), we will call this combined spectral shape the Combi spectrum.

The need to have both the types of behavior in a single parameterization used for testing and calibration follows from the discussions of Part I. The $f^{-4}$ behavior of the equilibrium interval is consistent both with observations (e.g., Donelan et al. 1985, hereafter DHH) and theory (e.g., Pushkarev et al. 2003). If, however, it is extended to $f_{\infty}=10 \mathrm{~Hz}$, it was found in Part I that such a spectrum cannot satisfy the principal constraint (2). Thus, the Phillips (1958) $f^{-5}$ equilibrium interval had to be reintroduced at higher frequencies. As mentioned in Part I, the existence of such an interval has also been confirmed by experiments, as well as the presence of both the subintervals in the single spectrum and the transition between them (e.g., Forristall 1981; Evans and Kibblewhite 1990; Kahma and Calkoen 1992; Babanin and Soloviev 1998a; Resio et al. 2004). Because of the cumulative breaking behavior, significant wave breaking is predicted at these frequencies; thus, the original Phillips' concept can be applied. According to this concept, if wave breaking dominates the dynamics and thus defines the spectral shape at certain scales, $f^{-5}$ behavior of the spectrum should be expected. Here, we will follow Kahma and Calkoen (1992), who found the transition frequency to be

$$
f_{t} \sim \frac{2.5}{\pi} \frac{g}{U_{10}},
$$

which is typically in the range of $f_{t} \sim 3 f_{p}$.

Figure 2 shows ratio of the residual $\Delta F(f)$ to the Combi spectrum $F(f)$ for the fully developed case of Fig. 1. The peak of the graph, for waves with relative frequency $f / f_{p} \sim$ 6 , corresponds to the highest relative rates of the waveenergy dissipation and is in agreement with observations (e.g., Gemmrich 2005). Figure 2 again highlights the nonzero dissipation at the spectral peak at full development and the need to identify values and behavior of the coefficients $a$ and $b$ in (4).

\section{a. Determining levels of the inherent and cumulative terms}

The coefficients $a$ and $b$ were introduced by Babanin and Young (2005) and Young and Babanin (2006) based on a single extreme-breaking record analyzed. They obtained $a=0.0065$ and assumed the same value for $b$; however, in a general case this issue obviously needs to be revisited.

Preliminary computations of the spectral dissipation function (4) were performed for the Combi spectrum of 
moderately forced waves with $U_{10} / c_{p}=2.7$ and wind speed $U_{10}=10 \mathrm{~m} \mathrm{~s}^{-1}$ (here, $c_{p}$ is the phase speed of the spectral-peak waves). Coefficients $a$ and $b$ were assumed $a=b=0.0065$. Results of these computations are presented in Fig. 3. The inherent breaking term $T_{1}(f)$ and the forced dissipation term $T_{2}(f)$ are also shown. The shape of the wave dissipation source function is the result of the superposition of the terms $T_{1}(f)$ and $T_{2}(f)$ [see Eq. (4)].

As implied in (4), the long-scale waves down to the size of dominant waves do not experience the induced dissipation. The contribution of the forced dissipation to the total increases toward higher frequencies until it saturates. This gradual transition is due to the integral of the forced dissipation term $T_{2}(f)$ in (4).

Obviously, the high-frequency waves, which have not reached under the saturated dissipation limit mostly experience forced dissipation because of the influence of longer waves, and their inherent breaking dissipation can be neglected. The coefficients $a$ and $b$ are crucial in determining the saturation level as well as the relative contributions of the two different types of dissipation. Therefore, to achieve the correct level of wave dissipation in the model, it is necessary to calibrate these coefficients carefully.

As mentioned in section 2, the major constraint for the dissipation function (4) is the condition (3). The ratio of the dissipation integral to the input integral was adopted from the experiment of Donelan (1998),

$$
R=\frac{\int S_{\mathrm{ds}}(f) d f}{\int S_{\text {in }}(f) d f} .
$$

According to Donelan (1998), this ratio stays within the range of $95 \%-100 \%$ for most stages of wave development, reaching $100 \%$ at the Pierson-Moskowitz limit. It is only at very early stages that the total wind input can be significantly larger than the total dissipation.

Donelan (1998) did not provide an explicit quantitative dependence for the ratio $R$; for practical purposes,

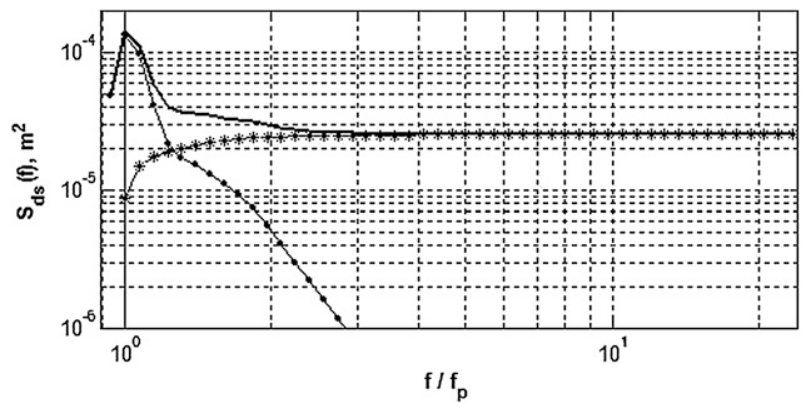

FIG. 3. Spectral dissipation function $S_{\mathrm{ds}}(f)$ [Eq. (4); bold line] with the terms $T_{1}(f)$ (line with dots) and $T_{2}(f)$ (line with asterisks) with coefficients $a=b=0.0065$. The computations were performed for the Combi spectrum with $f_{p}=0.13 \mathrm{~Hz}, U_{10} / c_{p}=2.7$, and $U_{10}=10 \mathrm{~m} \mathrm{~s}^{-1}$

his Fig. 6 was segmented, digitized, and parameterized here as the following:

$$
R=\left\{\begin{array}{lr}
-0.12 U_{10} / c_{p}+1.52, & 4.5<U_{10} / c_{p} \leq 5.8 \\
0.0031 U_{10} / c_{p}+0.96, & 1.5<U_{10} / c_{p} \leq 4.5 \\
-0.052 U_{10} / c_{p}+1.043, & 0.83<U_{10} / c_{p} \leq 1.5 \\
1, & U_{10} / c_{p} \leq 0.83
\end{array}\right.
$$

The upper wind-forcing limit of Donelan (1998) was $U_{10} / c_{p}=4.5$, and parameterization (15) also includes the range of very young dominant waves $U_{10} / c_{p}=4.5-5.8$. For this range of wave ages, the dissipation ratio $R$ was determined on the basis of consistency between the model results for the variance of the energy density spectra and the experimental data of Babanin and Soloviev (1998b). It was found that for very young waves the dissipation ratio is relatively small compared to the ratio for mature waves. For very young waves of $U_{10} / c_{p}=5.8$, it was of order $R=$ 0.82 (for more details, see section 4.3 of Tsagareli 2008).

The segmented parameterization (15) produces discontinuities of the derivatives, and such sharp transitions are undesirable in numerical modeling. Therefore, for application within a spectral model, the relationship was smoothed and used in this study in the following form:

$$
R_{\text {smooth }}=\left\{\begin{array}{lc}
0.97-0.07 \times\left(1+\tanh \left[3\left(U_{10} / c_{p}-5.2\right)\right]\right), & 2<U_{10} / c_{p} \leq 5.8 \\
0.97+0.015 \times\left(1-\tanh \left[5\left(U_{10} / c_{p}-1.1\right)\right]\right), & 0.9<U_{10} / c_{p} \leq 2, \\
1, & 0.83 \leq U_{10} / c_{p} \leq 0.9
\end{array}\right.
$$

The performance of (16) is demonstrated in Fig. 4. There are three stages with distinctly different behavior of the dissipation ratio. For very young waves and for well-developed waves $\left(U_{10} / c_{p}>4.5\right.$ and $U_{10} / c_{p}<1.5$, respectively), the ratio $R$ increases as waves mature; in between, it is relatively constant at approximately $96 \%-97 \%$.

The physical constraints (2) and (3) can be combined to calibrate the dissipation function (4): 


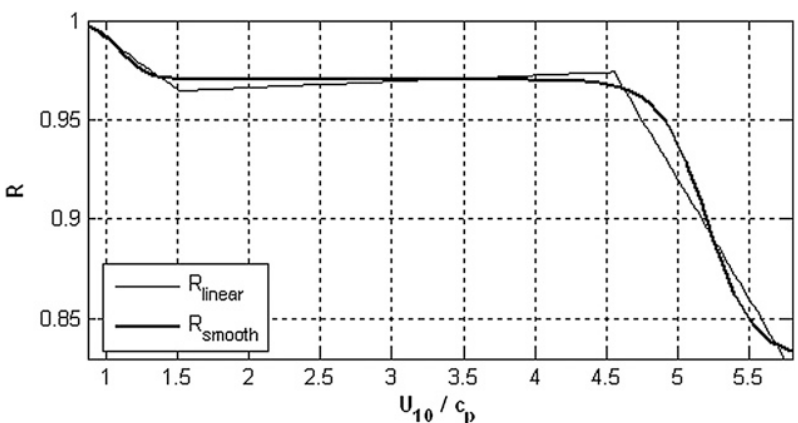

FIG. 4. Two parameterized forms of the dissipation ratio: $R_{\text {linear }}$ [Eq. (14)] and $R_{\text {smooth }}$ [Eq. (15)]. Computations were performed for the Combi spectra at the different stages of wave development $U_{10} / c_{p}$ for wind speed $U_{10}=10 \mathrm{~m} \mathrm{~s}^{-1}$.

$$
\int S_{\mathrm{ds}}(f) d f=R \int S_{\text {in }}(f) d f
$$

Because of the two-phase behavior of the spectral dissipation, the induced dissipation term $T_{2}$ of formulation (4) is zero below the peak; thus, the entire dissipation in this region was attributed to the inherent breaking to estimate the coefficient $a$ (note that this is an upper estimate of the dissipation because the nonlinear transfer could have contributed toward spectrum growth below the peak). As the waves approach full developments, the dissipation $T_{1}\left(f<f_{p}\right)$ becomes negligible and therefore $a \rightarrow 0$. Once the coefficient $a$ is calibrated, the quantitative dependence for $b\left(U / c_{p}\right)$ can be obtained based on the constraint (17).

The behavior of the coefficients $a$ and $b$ obtained this way is shown in Fig. 5 as a function of the wave-development stage $U_{10} / c_{p}$. The magnitude of these coefficients is compared with the experimental value $a_{\text {exp }}=0.0065$ estimated by Young and Babanin (2006).

As shown in the figure, values of both coefficients decrease as waves develop, and the rate of decrease accelerates as the waves approach full development. This trend, in particular, indicates a reduction of the relative wave-energy dissipation for the dominant waves as the wave system develops.

For young waves $\left(U_{10} / c_{p}>2\right)$, the coefficients $a$ and $b$ differ by an order of magnitude, with $a$ being larger. As can be seen in Fig. 6, the cumulative term, the magnitude of which is effectively determined by the coefficient $b$, dominates the dissipation at smaller scales of the wave spectrum (i.e., the tail of the dissipation function saturates because of the cumulative term, according to Fig. 3). This highlights the significance and importance of the cumulative term $T_{2}$, which, no matter how small it is in absolute value, cannot be disregarded in the spectral sense.

For older waves, the difference between the coefficients reduces; for a mature stage of development, $U_{10} / c_{p} \sim 1.2$,

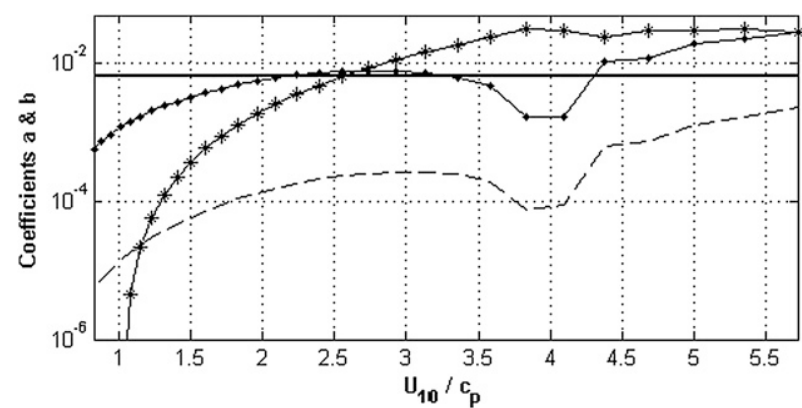

FIG. 5. Coefficients $a$ (line with asterisks) and $b$ (dashed line) as functions of the wind forcing parameter $U_{10} / c_{p}$ computed for the Combi spectra at wind speed $U_{10}=10 \mathrm{~m} \mathrm{~s}^{-1}$. The line with dots shows coefficient $b_{0}$ after the frequency correction was applied (section 4b). The experimental coefficient $a_{\exp }=0.0065$ (Young and Babanin 2006) is shown with a bold line.

the relationships cross: $a=b$. For even older waves, approaching full development the magnitude of the coefficient $a$ drops rapidly, signifying absence of wave breaking at the spectral peak (Banner et al. 2000). The coefficient $b$ continues to decrease gradually but unlike $a$ does not become zero. Even at these stages of development, the induced dissipation persists. This is due to, for example, stretching and compressing of short waves by underlying longer waves, thus causing these shorter waves to break (Donelan 2001).

Figure 5 also compares the coefficient $a$ obtained by means of the constraint (17) and experimental data (bold line). The only experimental estimate of $a=$ 0.0065 is available from Young and Babanin (2006) based on their analysis of a single wave record when wind forcing was quite extreme, $U_{10} / c_{p} \sim 6.5$. In Fig. 5, the value of $a=0.0065$ is achieved at $U_{10} / c_{p}=2.6$; at higher values of wind forcing, the magnitude is somewhat greater. Qualitatively, this is consistent with the experiment. Young and Babanin (2006) stressed that their estimate is a lower-bound approximation of the actual value because they measured the dissipation by comparing the difference in energy of wave trains that were already breaking to wave trains that had completed breaking and were once again gaining energy from the wind. By definition, this approach will underestimate the energy loss. In this regard, the quantitative agreement between the calibrated values of $a$ and the measurement is encouraging.

Figure 6 shows the dissipation source function $S_{\mathrm{ds}}(f)$ [Eq. (4)] based on the coefficients $a$ and $b$, computed for the Combi spectrum (12) with $U_{10} / c_{p}=2.7$ and wind speed $U_{10}=10 \mathrm{~m} \mathrm{~s}^{-1}$. The figure also shows the corresponding wind-energy-input source function $S_{\text {in }}(f)$ computed for the same wind-forcing conditions (Part I). The integrals of the two source functions are consistent 


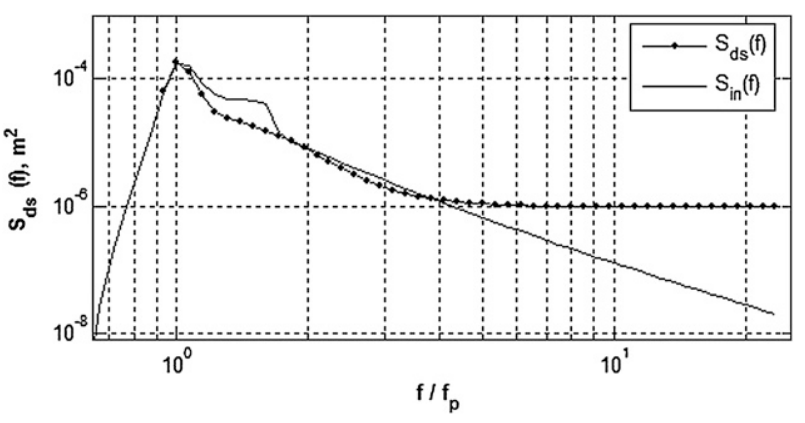

FIG. 6. The spectral dissipation function $S_{\mathrm{ds}}(f)$ (line with dots) with coefficients $a$ and $b$ of Fig. 5. Computations were performed for the Combi spectrum with wind forcing $U_{10} / c_{p}=2.7$ and wind speed $U_{10}=10 \mathrm{~m} \mathrm{~s}^{-1}$. The corresponding wind input $S_{\text {in }}(f)$ is shown as the plain line.

according to the physical constraint (17), but the shape of the dissipation function at high frequencies raises further questions about the calibration of $S_{\mathrm{ds}}(f)$.

The most striking feature of the comparison of $S_{\mathrm{ds}}(f)$ and $S_{\text {in }}(f)$ is the difference at the high frequencies, where the cumulative term $T_{2}(f)$ dominates, by up to 2 orders of magnitude. Mathematically, this feature is apparent if coefficients $a$ and $b$ in (4) are frequency independent (i.e., only vary as a function of wave age, as shown in Fig. 5). Physically, however, such a difference is difficult to justify because, to maintain the high dissipation rates, a very strong energy flux from the lower-frequency part of the spectrum by means of, for example, the nonlinear interaction term in (1) would be necessary.

This raises a question as to whether the coefficient $b$ is scale independent. It is likely that a frequency-dependent correction is required to ensure the magnitude of the wave dissipation $S_{\mathrm{ds}}(f)$ remains comparable with the wind-input function $S_{\text {in }}(f)$.

\section{b. Frequency-dependent coefficients $a$ and $b$}

To define the frequency-dependent form for the coefficient $b$, it was decided to apply a dimensionless correction function $Z(f)$, which ensured that the resulting magnitudes of the wave dissipation and wind-energyinput source functions are comparable in magnitude, $S_{\mathrm{ds}}(f) \sim S_{\mathrm{in}}(f)$. Note that this correction is only approximate because the nonlinear transfer should also play some role, as mentioned in section 4a. It should be pointed out that such a correction does not affect the principal physical constraint (17) because it is applied in the frequency region where values of the dissipation function are 2 or more orders of magnitude less than the peak and therefore their contribution into the integral is negligible.
A function of the following form was chosen:

$$
Z(f)=\left(\frac{f}{f_{p}}\right)^{\mu},
$$

where $\mu$ is the exponent fitted to the high-frequency tail of the wind-input source term $S_{\text {in }}(f)$ at each stage. The resulting coefficient for the cumulative dissipation term $T_{2}(f)$ can now be represented as

$$
b=b_{0} Z(f) \text {. }
$$

The magnitude of the coefficient $b_{0}$ computed at different stages of wave development are shown in Fig. 5 by the line with dots. These new values of $b_{0}$ are now significantly larger than the previous values of $b$ (dashed line). They are also closer to the experimental value $a_{\exp }$, which is what was also implied for the coefficient $b$ in the experimental paper by Young and Babanin (2006).

This new coefficient was tested for different types of wave spectra and for different wind speeds $U_{10}=7,10$, 15,20 , and $30 \mathrm{~m} \mathrm{~s}^{-1}$. Figure 7 demonstrates results of these computations at different stages of wave development $U_{10} / c_{p}$, where the top panel shows the results for JONSWAP spectra, the middle panel shows results for DHH spectra, and the bottom panel shows results for Combi spectra (12).

Values of coefficient $b_{0}$ computed for DHH spectra (Fig. 7b) are much smaller in magnitude than those for JONSWAP and Combi spectra. The coefficient $b_{0}$ in the figure is obviously wind dependent, in contrast to the coefficient $a$, which is almost the same in all subplots. The dependence of $b_{0}$ on the wind speed $U_{10}$ is particularly strong for young waves. As waves approach full development, this wind speed dependence becomes negligible.

Figure 7 also reveals some problems of the frequency correction to coefficient $b_{0}$. At light winds and young waves, the coefficient is negative, corresponding to a positive flux of energy to short-scale waves in such circumstances. Given the many assumptions involved, it is most likely that the negative dissipation is an artifact of the functional form of the selected correction factor.

Once the constraint (17) is applied to the dissipation formulation (4), the coefficients $a$ and $b$ are no longer independent, as explained earlier. To keep the cumulative term positive, an upper limit was imposed on the coefficient $a$ such that the condition of $b>0$ is always satisfied. In technical terms, the correction function $Z(f)$, was applied to coefficient $a$ in the high-frequency range only: 

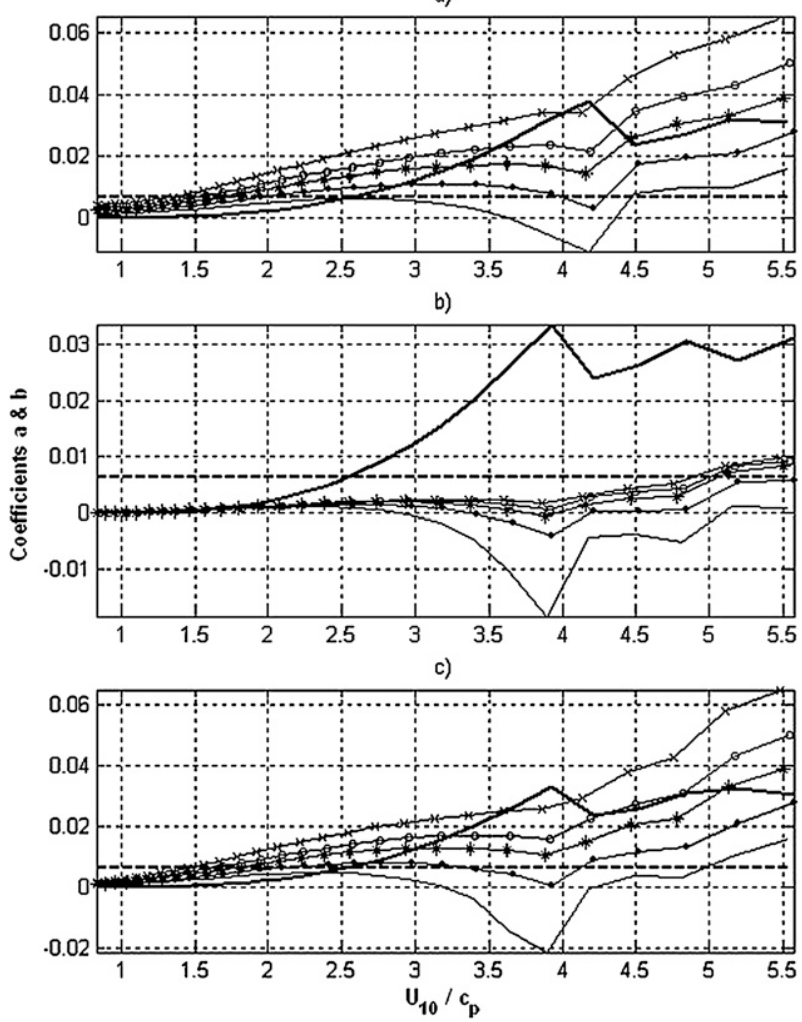

FIG. 7. Results of computations of coefficients $a$ (bold line in all subplots) and $b_{o}$ [Eq. (19)] for (a) JONSWAP, (b) DHH spectra, and (c) Combi spectra as a function of inverse wave age $U_{10} / c_{p}$ at different wind speeds $U_{10}=7$ (plain line), 10 (line with dots), 15 (line with asterisks), 20 (line with circles), and $30 \mathrm{~m} \mathrm{~s}^{-1}$ (line with crosses). The experimental coefficient $a_{\exp }=0.0065$ (bold dashed line) is shown.

$$
a= \begin{cases}a_{0}, & f \leq f_{p}, \\ a_{0} Z(f), & f>f_{p} .\end{cases}
$$

As a result of this correction, values of the coefficient $a$ decreased somewhat and coefficient $b$ correspondingly increased and now stays positive in all circumstances.

Figure 8 (top) shows the wave dissipation source function $S_{\mathrm{ds}}(f)$ with the frequency-corrected coefficients $a$ and $b$ according to (19) and (20) at different stages of wave development $U_{10} / c_{p}$. At intermediate wind forcing of $U_{10} / c_{p}=2.7$, the dissipation exceeds values of the wind input in the spectral tail, which implies an additional influx of energy resulting from nonlinear transfer in this spectral region. Other than this region, the dissipation is always smaller than the input across the spectrum.

In Fig. 8 (bottom), the spectral dissipation function is shown computed for $U_{10} / c_{p}=2.7$ at different wind speeds of $U_{10}=7,10,15,20$, and $30 \mathrm{~m} \mathrm{~s}^{-1}$. The figure clearly demonstrates growth of the dissipation level as (a)

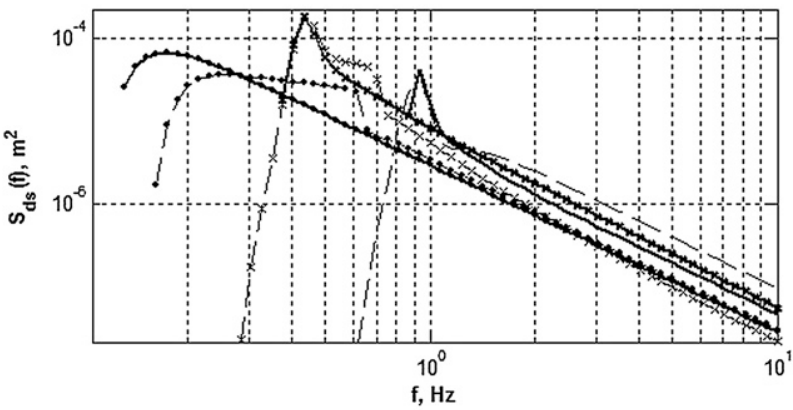

(b)

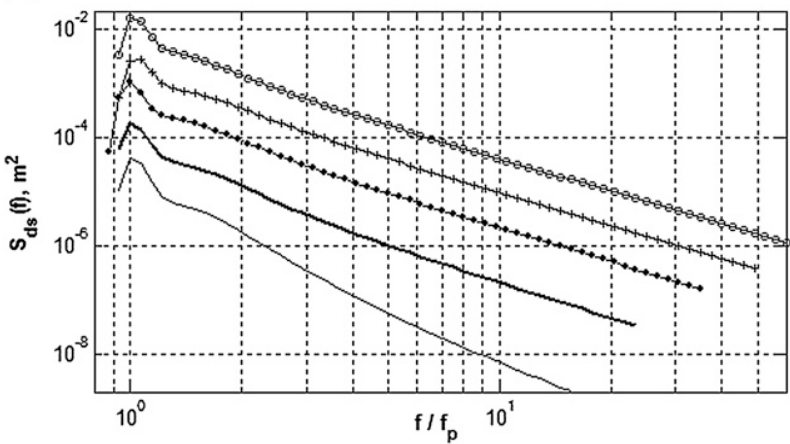

FIG. 8. The spectral dissipation source function $S_{\mathrm{ds}}(f)$ [Eq. (4)] computed with coefficients $a$ [Eq. (20)] and $b$ [Eq. (19)]. Computations were performed for the Combi spectra. (top) Different stages of the wave development $U_{10} / c_{p}=5.7$ (bold line), 2.7 (bold line with crosses), and 0.83 (bold line with dots) for the wind speed $U_{10}=10 \mathrm{~m} \mathrm{~s}^{-1}$. Respective wind-input source functions $S_{\text {in }}(f)$ are also shown with plain lines marked with symbols corresponding to the dissipation functions. (bottom) Waves at $U_{10} / c_{p}=2.7$ for the wind speeds $U_{10}=7$ (plain line), 10 (bold line), 15 (line with dots), 20 (line with crosses), and $30 \mathrm{~m} \mathrm{~s}^{-1}$ (line with circles).

the wind speed increases. Furthermore, the contribution of the forced (induced) cumulative wave breaking to the total wave dissipation slightly increases with increasing wind speed as indicated by the reduction of the slope of the high-frequency tail of the wave dissipation function.

\section{c. Directional spreading of the dissipation function}

In the present study, an attempt has been made to model the least known feature of the spectral dissipation function, its directional behavior. Previously, isotropic or unimodal directional shapes were assumed for the dissipation source term. However, the recent experiment at Lake George (Young and Babanin 2006) revealed that the dissipation function may have symmetric maxima at angles oblique to the main wave-propagation direction. In terms of spectral modeling, this fact can be interpreted as a bimodal shape of the directional spreading for the dissipation function. Note that this is a feasibility study only, which is intended to describe how the directional spreading of the wave spectrum can potentially be controlled 
through directionality of the dissipation term. These moderate alterations of directional spreading dissipation features do not impact the frequency-distributed behavior of the source terms in any way.

Here, the directional spreading function was developed as a superposition of two Gaussian functions. According to the experimental results of Young and Babanin (2006), the angle of separation between the maxima and the main wave-propagation direction can vary. Therefore, the new directional function for the dissipation term was provided with sufficient flexibility to modify the shape in both directional and frequency spaces. The new directional spreading function includes the ability to 1) symmetrically shift the locations of the peaks in direction, 2) vary the magnitude of the trough between the maxima, 3 ) vary the cross-sectional shapes as a function of frequency, and 4) vary with different wind-forcing conditions.

The spreading function $V$ is assumed to be of the form

$$
V\left(\theta, f, U_{10} / c_{p}\right)=\left\{\begin{array}{ll}
V_{1}\left(\theta, f, U_{10} / c_{p}\right)=A(f) \exp \left(-p\left(\theta+\theta_{p}\right)^{2}\right), & \theta<0 \\
V_{2}\left(\theta, f, U_{10} / c_{p}\right)=A(f) \exp \left(-p\left(\theta-\theta_{p}\right)^{2}\right), & \theta \geq 0
\end{array},\right.
$$

where $p=p\left(f, U_{10} / c_{p}\right)$ is the parameter that determines the depth of the middle trough, $\theta$ is the angle (in radians) relative to the main propagation direction of the waves, and $\theta_{p}=\theta_{p}\left(f, U_{10} / c_{p}\right)$ is the angle (in radians) of the maximum dissipation rates relative to the main propagation direction of the waves.

The term $A(f)$ is the inverse integral of the directional spread defined in (5). To avoid confusion, however, it has to be stressed that the directional wave-spectrum spreading function and the directional dissipation spreading function are different properties. For clarity, the normalized dissipation directional spread will be designated $D$ rather than $K$, which is reserved for the function of normalized wave directional spectrum in (5); that is, we used $D(f, \theta)=\exp \left(-p\left(\theta \pm \theta_{p}\right)^{2}\right)$ in (21). The normalization condition $\int_{-\pi}^{\pi} V(f, \theta) d \theta=1$ is then satisfied; if the angle $\theta_{p}=0$, the directional spreading has a unimodal shape.

The functional dependence of parameters $p\left(f, U_{10} / c_{p}\right)$ and $\theta_{p}\left(f, U_{10} / c_{p}\right)$ in (21) on frequency and wind forcing was not obtained explicitly in this study. Rather, it was determined on the basis of the consistency of modeled wave growth and experimental data. As the angle $\theta_{p}$ increases, the lobes of the directional spreading function move farther apart, enhancing the depth of the trough between them. Increasing the parameter $p$ reduces the width of the lobes, also increasing the depth of the trough. As a result, variations of parameters $p$ and $\theta_{p}$ lead to different directional spreading of the dissipation, which allows the model to control the directional wave spectrum, a property that has proved difficult in previous modeling tests (e.g., Banner and Young 1994). Figure 9 demonstrates the resulting directional spreading function $V\left(\theta, f, U_{10} / c_{p}\right)$ [Eq. (21)] with different values for the parameters $p$ and $\theta_{p}$. For convenience of comparison, all the directional spreading functions were normalized by the maximum value at the angle $\theta_{p}$ [i.e., $D=V\left(\theta_{p}, f\right.$, $\left.\left.U_{10} / c_{p}\right)=1\right]$.
Figure 10 shows an initial model setup of the twodimensional spectral dissipation function $S_{\mathrm{ds}}(f, \theta)$ with the bimodal directional spreading function $V\left(\theta, f, U_{10} / c_{p}\right)$ [Eq. (21)]. For the angle $\theta_{p}\left(f, U_{10} / c_{p}\right)$, initially, a linear dependence was assumed:

$$
\left\{\begin{array}{l}
\theta_{p}(f)=\theta_{p}\left(f_{p}\right)+\Delta \theta_{f} \frac{\left(f-f_{s}\right)}{\left(f_{\text {cut }}-f_{s}\right)} \\
\theta_{p}\left(U_{10} / c_{p}\right)=\theta_{p}\left(f_{p}\right)+\Delta \theta_{\text {ucp }} \frac{\left(5.7-U_{10} / c_{p}\right)}{(5.7-0.83)}
\end{array}\right.
$$

where $\Delta \theta_{f}$ is the total directional span of the peak angle over the frequency scale, $\Delta \theta_{\text {ucp }}$ is the total increment in the course of the wave evolution, and $f_{s}$ is the lowest frequency where the bimodal spreading is observed. In the figure, $\theta_{p}=20^{\circ}, p=1$, and the initial setup was done for the Combi spectrum with $U_{10} / c_{p}=2.7$ and $U_{10}=$ $10 \mathrm{~m} \mathrm{~s}^{-1}$. A distinct trough in the dissipation $S_{\mathrm{ds}}(f, \theta)$ is visible in the main direction of the wave propagation at $\theta=0^{\circ}$. At the upper frequency $f_{\text {cut }}$, the source function peaks are located symmetrically to the main propagation direction at the angle $\theta_{p}=30^{\circ}$. Thus, the parameters of the directional function (21) and (22) allow us to vary the shape of the two-dimensional dissipation function $S_{\mathrm{ds}}(f, \theta)$, which is then allowed to evolve in the course of wave evolution, as described in section 5 .

\section{Testing the dissipation function}

Once the experimental properties of the dissipation function (4) were defined and calibrated, it was used in the numerical simulations and the results were compared with available experimental estimates. The only experimental data suitable for direct comparisons are those obtained, based on dimensional considerations, by means of estimating distributions of the length of breaking crests. This is done in section $5 \mathrm{a}$. 


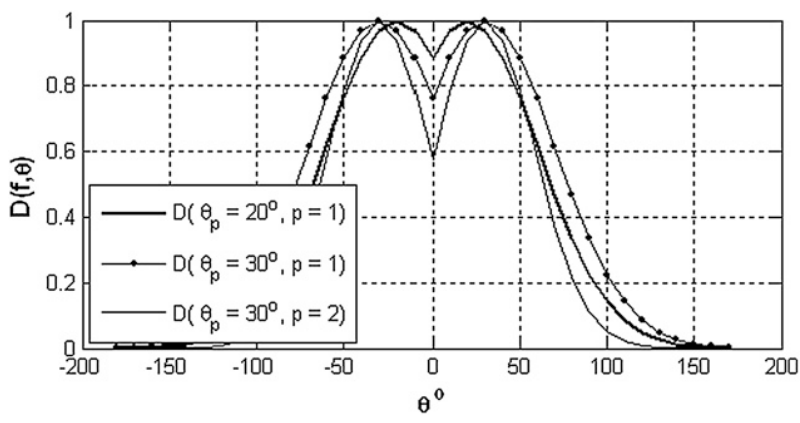

FIG. 9. Normalized directional spreading function $V\left(f, \theta_{p}\right)$ [Eq. (21)] denoted as $D$ with different values of the parameters $\theta_{p}$ and $p$.

In section $5 b$, wave-evolution tests conducted with the model WAVETIME, which employs full computations of the nonlinear integral (van Vledder 2002, 2006), are described. It has to be stressed again that, unlike the majority of similar exercises conducted over the years, in this study the dissipation function was calibrated first and then verified in the model. In previous studies, the model tests were used to calibrate the dissipation rather than validate its performance.

\section{a. Comparisons with other measurements}

A property of whitecapping that can be measured and converted into a spectral dissipation term based on dimensional arguments (Duncan 1981) is $\Lambda(c)$, the average length of breaking crests per unit area per unit interval of phase speed $c$ (Phillips et al. 2001). A significant number of attempts to measure this quantity have recently been undertaken, including Melville and Matusov (2002) and Gemmrich (2005).

Melville and Matusov (2002) obtained the spectral distribution of $\Lambda(c)$ experimentally as a function of $c$,

$$
\Lambda(c)\left(\frac{10}{U_{10}}\right)^{3}=3.3 \times 10^{-4} e^{-0.64 c},
$$

which can then be converted into a dissipation function

$$
\begin{aligned}
& S_{\mathrm{ds}}(c)=b_{b r} \rho_{w} g^{-1} c^{5} \Lambda(c)\left(\frac{10}{U_{10}}\right)^{3} \text { and } \\
& S_{\mathrm{ds}}(f)=\frac{g}{2 \pi} \frac{1}{f^{2}} S_{\mathrm{ds}}(c) .
\end{aligned}
$$

Here, $\rho_{w}$ is the water density, $g$ is the gravitational constant, and $b_{b r}$ is an empirical parameter [routinely named just parameter $b$, but here the subscript is used to distinguish it from the property that defines the level of the cumulative term $T_{2}(f)$ in (4)]. In the case of a steady breaker, $b_{b r}$ is simply a proportionality coefficient. However, in the field the breaking is principally unsteady. Measurements within realistic unsteady-breaking conditions in the laboratory and the field indicate $b_{b r}$ varies by

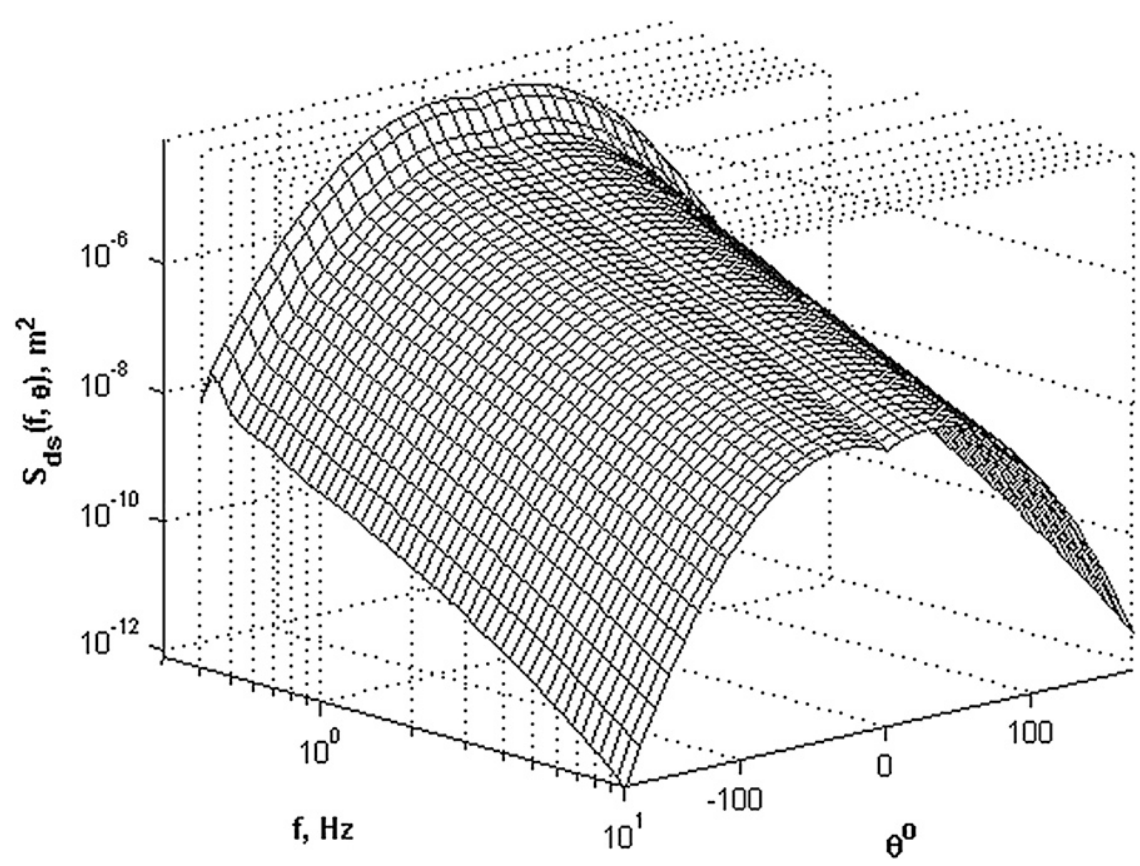

FIG. 10. Two-dimensional dissipation function $S_{\mathrm{ds}}(f, \theta)$ with the bimodal directional spreading $V\left(f, \theta_{p}\right)[$ Eqs. (21) and (22)] as described in section 4c. 
up to 4 orders of magnitude (for more details, see, e.g., Babanin 2009). Some studies point out its dependence on wave steepness (e.g., Melville and Matusov 2002; Drazen et al. 2008), wheras others argue that it is not the average steepness but rather local variations of the steepness that are important (i.e., wave slope, crest-to-wavelength ratio). In addition, properties such as the density of the whitecapping foam and the relative orbital velocity (with respect to the phase speed) may also play a part in determining $b_{b r}$ (Gemmrich et al. 2008).

The connection between dissipation in a breaking wave with its phase speed is clearly not applicable in the case of induced breaking (i.e., the breaking of short waves caused and carried by the large waves). Therefore, it is only the inherent term of dissipation (4) that should be considered in (25) for comparison purposes. Thus, a dissipation formulation of the type shown in (24) is strictly only applicable at the spectral peak region, where the cumulative effect is negligible.

Therefore, to reduce the uncertainty, values of coefficient $b_{b r}$ were compared at the spectral peak. The spectral dissipation function $S_{\mathrm{ds}}(f)$ [Eq. (4)] was computed for the modeled Combi spectrum (12) of mature waves with $U_{10} / c_{p}=1.3$ and at the wind speeds of $U_{10}=$ $7.2,9.8$, and $13.6 \mathrm{~m} \mathrm{~s}^{-1}$ (i.e., as in Melville and Matusov 2002), and it was compared with the dissipation in (23)(25). Reasonable agreement was achieved at $b_{b r} \approx 0.01$ (see Fig. 11). This quantity is close to the value $b_{b r}=$ $8.5 \times 10^{-3}$ used by Melville and Matusov (2002).

In Fig. 11, the parameter $b_{b r}$ of (24) is plotted as a function of inverse wave age $U_{10} / c_{p}$ (solid line), obtained from the dissipation function (4) at the spectral peak of the Combi spectrum (12) for different wind speeds: $U_{10}=7.2$ (plain line), 9.8 (line with dots), 13.6 (line with asterisks), 15 (line with circles), 20 (line with crosses), and $30 \mathrm{~m} \mathrm{~s}^{-1}$ (line with diamonds). The straight solid line is the value obtained in Melville and Matusov (2002).

Even at the peak, where the induced breaking is absent and the dimensional hypothesis of Duncan (1981) can be expected to hold, the proportionality coefficient varies by many orders of magnitude depending on the wave age and, for the same wave age, on the wind speed. It is only in the range of the wind forcing conditions $1.5<U_{10} / c_{p}<2$ that the coefficient $b_{b r}$ is close to the value of $8.5 \times 10^{-3}$ of Melville and Matusov (2002) for most wind speeds.

For wind speeds $U_{10} \leq 20 \mathrm{~m} \mathrm{~s}^{-1}$, as waves develop the coefficient $b_{b r}$ exhibits a reducing trend reaching a value on the order of $10^{-3}$ at full development $\left(10^{-2}\right.$ at $U_{10} \sim$ $\left.20 \mathrm{~m} \mathrm{~s}^{-1}\right)$. For very strong winds, $U_{10}=30 \mathrm{~m} \mathrm{~s}^{-1}$ in the figure, the coefficient $b_{b r}$ exhibits significant growth

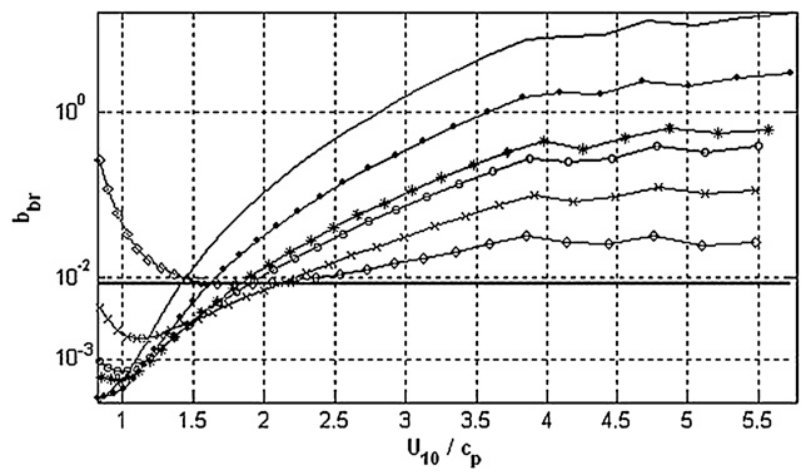

FIG. 11. Dependence of the parameter $b_{b r}$ [Eq. (24)] on the sea state $U_{10} / c_{p}$, based on the dissipation function (4), computed at the spectral peak of the Combi spectra for different wind speeds: $U_{10}=$ 7.2 (plain line), 9.8 (line with dots), 13.6 (line with asterisks), 15 (line with circles), 20 (line with crosses), and $30 \mathrm{~m} \mathrm{~s}^{-1}$ (line with diamonds). The straight solid line is the value obtained in Melville and Matusov (2002).

toward full development after it reached a minimum close to the Melville-Matusov value.

Comparison of the dissipation function (4) with the dissipation function based on the phase speed $c$ only [Eq. (24)] is shown in Fig. 12. For convenience, both functions were converted into wavenumber space, and the dissipation (4) was weighted by $\left(10 / U_{10}\right)^{3}$. Computations are performed for the Combi spectra with $U_{10} / c_{p}=1.3$ at wind speeds of $U_{10}=7.2,9.8$, and $13.6 \mathrm{~m} \mathrm{~s}^{-1}$. For the dissipation in (24), different values of the coefficient $b_{b r}=$ $2 \times 10^{-5}, 1 \times 10^{-3}$, and $8.5 \times 10^{-3}$ are employed.

As has already been pointed out by Babanin and Young (2005) and Babanin et al. (2007c), (24) is expected to underestimate the dissipation at smaller scales away from the spectral peak because, even if there was a universal constant value for the proportionality coefficient $b_{b r}$, it would only be applicable to the inherent dissipation term $T_{1}$ of the total dissipation in (4), whereas the term $T_{2}$ will tend to dominate at those scales. This is clearly the case in the figure.

At this stage of wave development $\left(U_{10} / c_{p}=1.3\right)$, agreement of the two dissipations at the spectral peak is reasonable for the Melville-Matusov value of $b_{b r}=$ $8.5 \times 10^{-3}$. The value of $b_{b r}=2 \times 10^{-5}$ suggested by Gemmrich (2005) gives much lower dissipation values across the entire spectral band. As discussed by Tsagareli (2008), however, there is a dimensional issue in comparing the $b_{b r}$ coefficients of Melville and Matusov (2002) and Gemmrich (2005) because of the normalization by $\left(10 / U_{10}\right)^{3}$. If this issue is accounted for, the agreement between the experimental outcomes of the two studies and the peak of the dissipation function (4) at $U_{10} / c_{p}=1.3$ is quite good (not shown here). 


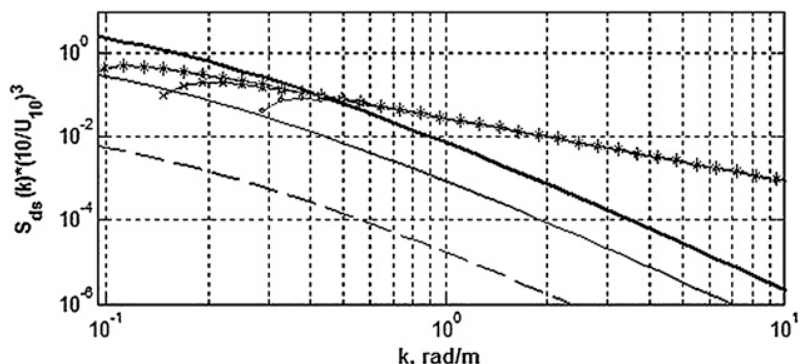

FIG. 12. Comparison of the dissipation functions (4) and (24), both converted into wavenumber space. The dissipation [Eq. (4)] is weighted by $\left(10 / U_{10}\right)^{3}$, and computations are performed for the Combi spectra at $U_{10} / c_{p}=1.3$ for the wind speeds $U_{10}=7.2$ (line with dots), 9.8 (line with crosses), and $13.6 \mathrm{~m} \mathrm{~s}^{-1}$ (line with asterisks). For dissipation [Eq. (24)], different values of coefficient $b_{b r}=$ $2 \times 10^{-5}$ (dashed line), $1 \times 10^{-3}$ (plain line), and $8.5 \times 10^{-3}$ (bold line) are employed.

\section{b. Modeling the spectral evolution using the new wind-input and whitecapping dissipation functions}

The numerical modeling consisted of a series of simulations of duration-limited evolution of wind waves, conducted using the third-generation one-dimensional research wave model WAVETIME developed by G. van Vledder (Alkyon, 2002, personal communication). It includes the Webb-Resio-Tracy (WRT) algorithm (Webb 1978; Tracy and Resio 1982; van Vledder 2002, 2006) for computation of the nonlinear wave interaction term.

WAVETIME solves RTE (1) and operates in one of four different modes: source mode, computations of the source terms for a given spectrum; time mode, computations of duration-limited growth; fetch mode, computations of fetch-limited growth; and grid mode, computations of wave propagation on a spatial grid. For the present study, only duration-limited conditions were considered. The model is able to perform computations using either explicit or implicit numerical schemes. One of the advantages of this model is its modular design, which enables further development of the model for research purposes (e.g., replacement of the default source functions with new forms).

A detailed technical account of the model and numerical setup used in the present study can be found in Tsagareli (2008). This description includes choice of the spectral grid, numerical scheme, optimal time step, and initial spectrum. It also describes validation of the model, including verification of $S_{\mathrm{nl}}$ and other default source terms.

Calibration of the wind-input term was conducted in Part I. The dissipation term was calibrated in section 4 for typical parametric spectral forms JONSWAP, DHH,

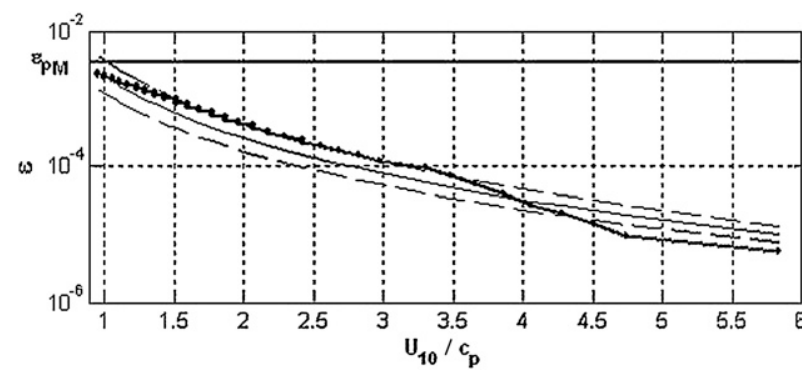

FIG. 13. Dependence of the total dimensionless wave energy $\varepsilon=\sigma^{2} g^{2} / U_{10}^{2}$ on the sea state $U_{10} / c_{p}$. The model results are compared with the experimental data of Babanin and Soloviev (1998b; plain line) with $10 \%$ approximation limits (dashed lines). The $\varepsilon_{\mathrm{PM}}=3.64 \times 10^{-3}$ of the Pierson-Moskowitz limit is also shown.

and Combi [Eq. (12)]. However, the calibrated forms were not imposed in the course of the simulations conducted here using WAVETIME. Instead, the source functions, within their functional forms, as well as the wave spectra, were allowed to evolve freely, provided that the principal constraints (2) and (17) are satisfied at each time step. If these constraints are not satisfied, the free parameters of the spectral shape of the source functions were adjusted dynamically. The only outside constraints at each stage of wave development were the wave-age-dependent wave-induced stress, which is independently obtained by means of the bulk formula of Guan and Xie (2004), and the ratio of the total input and dissipation according to Donelan (1998).

Figure 13 shows the integral dependence of the total dimensionless wave energy $\varepsilon=\sigma^{2} g^{2} / U_{10}^{2}$ on the sea state $U_{10} / c_{p}\left(\sigma^{2}\right.$ is wave variance: i.e., wave energy expressed in $\mathrm{m}^{2}$ ). The model results are compared with the experimental data of Babanin and Soloviev (1998b; plain line) with $10 \%$ approximation limits (dashed lines). The computations shown were conducted for $U_{10}=10 \mathrm{~m} \mathrm{~s}^{-1}$, but dimensionless results for $U_{10}=7,15$, and $20 \mathrm{~m} \mathrm{~s}^{-1}$ were similar (not shown here). The value $\varepsilon_{\mathrm{PM}}=3.64 \times$ $10^{-3}$ of the Pierson-Moskowitz limit is also shown.

Within the $10 \%$ limits, the agreement is good throughout the entire wave development. The modeled growth is somewhat faster than the parameterization at early stages of development and slower as full development approaches. The maximum difference between the model results and the observational data is at $U_{10} / c_{p} \sim 3.5-2$. In physical terms, such differences are expected (e.g., Badulin et al. 2007), particularly at the Pierson-Moskowitz limit. The parameterizations have a significant degree of uncertainty resulting from both the scatter of the experimental data points that they are based on and the practical difficulties of the actual observation of full development. 


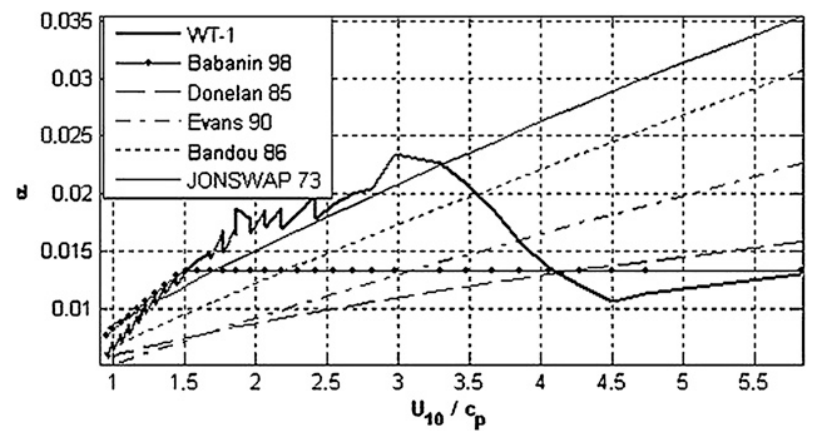

FIG. 14. Values of $\alpha$ as a function of $U_{10} / c_{p}$ at $U_{10}=10 \mathrm{~m} \mathrm{~s}^{-1}$ obtained by means of WAVETIME modeling (denoted as "WT-1"). The results of computations are compared with the experimental dependences obtained in different studies [Babanin and Soloviev 1998b; Donelan et al. 1985; Evans and Kibblewhite 1990; Bandou et al. 1986; Hasselmann et al. 1973 (JONSWAP)].

One of the main spectral characteristics of the wave spectrum, for validation purposes, is the tail level $\alpha$ in (12) and the respective shapes of the JONSWAP and DHH spectra. In Fig. 14, a number of parameterizations of $\alpha$ as a function of $U_{10} / c_{p}$ are shown for comparison with the model results at $U_{10}=10 \mathrm{~m} \mathrm{~s}^{-1}$.

Most of the parameterization, when extrapolated to high values of wind forcing $U_{10} / c_{p}$, show a continuous dependence for $\alpha$. Babanin and Soloviev (1998b), however, who conducted field measurements of both mature and very young waves, argued that $\alpha$ only varies for well-developed waves and otherwise stays constant and close to the Phillips (1966) value. The behavior of the spectral tail in the present simulations appear to obey this kind of segmented behavior (see Fig. 14) and also are in satisfactory agreement with other measurements in the range of their applicability.

Traditionally, the most difficult characteristics of wave evolution to reproduce by means of spectral wave modeling are the directional properties of wave fields (e.g., Banner and Young 1994). The bimodal directional shape of the dissipation spectrum described in section $4 \mathrm{c}$ increases the flexibility of the model to control this property of the wave spectrum: that is, if the wave directional spectrum is too broad, the bimodal directional distribution can be used to make it narrower and the degree of separation of the side lobes can be used to control the width of the wave directional spread. Otherwise, if it is too narrow, a unimodal dissipation will make it broader [i.e., if $\theta \approx \theta_{p}$ in (21)].

Figure 15 compares the WAVETIME model results for the directional spreading parameter $A$ [Eq. (5)], plotted as a function of wind forcing $U_{10} / c_{p}$, with the experimental data of Babanin and Soloviev (1998a). The computations were performed for the directional spectral slices at the frequencies $f_{p}, 2 f_{p}$, and $3 f_{p}$.

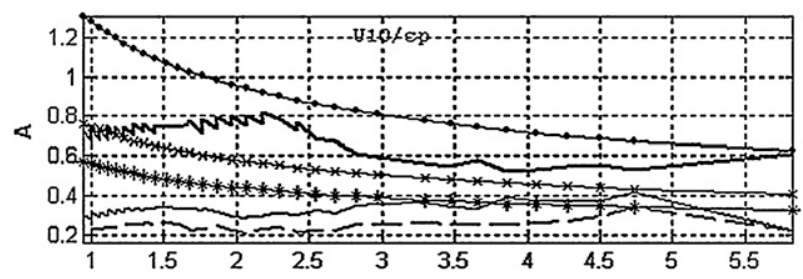

FIG. 15. Comparisons of the model results for the directional spreading parameter $A$ [Eq. (5)], plotted as a function of wind forcing $U_{10} / c_{p}$, with the experimental data of Babanin and Soloviev (1998a; lines with symbols). The computations were performed for the directional spectral slices at the frequencies $f_{p}$ (bold solid line and line with dots), $2 f_{p}$ (plain line and line with crosses), and $3 f_{p}$ (dashed line and line with asterisks).

The modeled evolution reproduces the reduction of the inverse directional width $A$ as a function of dimensionless frequency $f / f_{p}$ very well and also demonstrates a reasonable consistency with the wave-age evolution of the directional spread at the spectral peak. At the higher relative frequencies, although the quantitative agreement is reasonable, the decrease of the directional width is not picked up by the model. Addressing this issue is left for future investigation. The new directional shape of the dissipation function has a number of means to deal with this difficult problem and to control directional evolution of the wave spectrum through adjusting the directional shape and width of the dissipation angular function (21).

\section{Conclusions}

Part I was dedicated to testing and calibration of the new wind-input function (Donelan et al. 2006). This article is devoted to the new dissipation function (Babanin and Young 2005; Young and Babanin 2006).

Both the new wind input and dissipation are observation based; that is, they parameterize experimentally observed aspects presently not accounted for in operational source functions. In the case of the input term, these are its nonlinear behavior and its relative reduction at conditions of strong winds/steep waves. For the dissipation function, the main new features are the wave-breaking threshold and the cumulative term.

Although the source terms were developed based on observational data, these data covered only a limited range of environmental parameters. Because of experimental limitations, the direct measurements of windinput growth rates were confined to the region of the spectral peak. For the dissipation term, direct estimates of the magnitude of the inherent breaking and cumulative terms were conducted for only one wave record. Therefore, in Part I and here, substantial calibration of the wind input and dissipation across the spectrum and 
for different wind-forcing conditions were conducted, and further experimental and theoretical guidance is still needed.

The experimental dissipation of Babanin and Young (2005) and Young and Babanin (2006) is calibrated and tested based on measured physical constraints. These constraints consist of the wind stress, the value of which must match the integrated wind input at any stage of wave development, and the ratio of the integrated wind input and integrated wave-energy dissipation, which is also known experimentally as a function of wave age.

The approach adopted here is principally different to previous approaches, which have relied on tuning the various source terms to reproduce observed trends in wind-wave evolution. The introduction of these physical constraints in the calibration enables a more consistent description of the physics. The new technique allows us to test the dissipation source function (as well as the windinput function in Part I) separately before it is applied in the wave-evolution model in conjunction with other sources and sinks. The subsequent model runs are hence a validation of the source terms rather than a calibration.

The new dissipation $S_{\mathrm{ds}}$ was implemented in the one-dimensional wave model WAVETIME with exact nonlinear-term computations and verified against measured integral, spectral, and directional properties of wave field. The free parameters of the dissipation shape, although already calibrated, were not imposed (e.g., dependence of coefficients $a$ and $b$ on wave age) but were allowed to evolve in the course of wave evolution. The resulting evolution yields results consistent with previously observed parameters.

Acknowledgments. We are very grateful for the suggestions provided by Erick Rogers from the Naval Research Laboratory. We also acknowledge the operational support of Gerbrant van Vledder of Alkyon Hydraulic Consultancy and Research, the Netherlands. The authors acknowledge financial support through ONR Grant N00014-101-0418.

\section{REFERENCES}

Ardhuin, F., T. H. C. Herbers, G. P. van Vledder, K. P. Watts, R. Jensen, and H. C. Graber, 2007: Swell and slantingfetch effects on wind wave growth. J. Phys. Oceanogr., 37, 908-931.

Babanin, A. V., 2009: Breaking of ocean surface waves. Acta Phys. Slovaca, 59, 305-535.

, and Y. P. Soloviev, 1987: Parameterization of width of directional energy distributions of wind-generated waves at limited fetches. Izv. Akad. Nauk SSSR Fiz. Atmos. Okeana, 23, 645-651.

, and - , 1998a: Variability of directional spectra of windgenerated waves, studied by means of wave staff arrays. Mar. Freshwater Res., 49, 89-101.
- and - 1998b: Field investigation of transformation of the wind wave frequency spectrum with fetch and the stage of development. J. Phys. Oceanogr., 28, 563-576.

— , and I. R. Young, 2005: Two-phase behaviour of the spectral dissipation of wind waves. Proc. Fifth Int. Symp. Ocean Waves Measurement and Analysis, Madrid, Spain, ASCE, 51.

—_, and A. J. van der Westhuysen, 2008: Physics of "saturationbased" dissipation functions proposed for wave forecast models J. Phys. Oceanogr., 38, 1831-1841.

_ I. R. Young, and M. L. Banner, 2001: Breaking probabilities for dominant surface waves on water of finite constant depth. J. Geophys. Res., 106, 11 659-11 676.

_ M. L. Banner, I. R. Young, and M. A. Donelan, 2007a: Wave follower measurements of the wind input spectral function. Part III: Parameterization of the wind input enhancement due to wave breaking. J. Phys. Oceanogr., 37, 2764-2775.

, K. N. Tsagareli, I. R. Young, and D. Walker, 2007b: Implementation of new experimental input/dissipation terms for modeling spectral evolution of wind waves. Proc. 10th Int. Workshop on Wave Hindcasting and Forecasting and Coastal Hazards Symp., Oahu, HI, U.S. Army Engineer Research and Development Center, 12 pp.

_ I. R. Young, R. Manasseh, and E. Schultz, 2007c: Spectral dissipation term for wave forecast models, experimental study. Proc. 10th Int. Workshop on Wave Hindcasting and Forecasting and Coastal Hazards Symp., Oahu, HI, U.S. Army Engineer Research and Development Center, 19 pp.

Badulin, S. I., A. V. Babanin, V. E. Zakharov, and D. Resio, 2007: Weakly turbulent laws of wind-wave growth. J. Fluid Mech., 591, 339-378.

Bandou, T., H. Mitsuyasu, and T. Kusaba, 1986: An experimental study of wind waves and low frequency oscillations of water surface. Rep. Res. Inst. Appl. Mech., 33, 13-32.

Banner, M. L., and I. R. Young, 1994: Modeling spectral dissipation in the evolution of wind waves. Part I: Assessment of existing model performance. J. Phys. Oceanogr., 24, 1550-1571.

_ driven air-water interfaces. J. Fluid Mech., 364, 115-145.

_ I. S. F. Jones, and J. C. Trinder, 1989: Wavenumber spectra of short gravity waves. J. Fluid Mech., 198, 321-344.

— A. V. Babanin, and I. R. Young, 2000: Breaking probability for dominant waves on the sea surface. J. Phys. Oceanogr., 30, 3145-3160.

— J. R. Gemmrich, and D. M. Farmer, 2002: Multi-scale measurements of ocean wave breaking probability. J. Phys. Oceanogr., 32, 3364-3375.

Cavaleri, L., and Coauthors, 2007: Wave modelling-The state of the art. Prog. Oceanogr., 75, 603-674.

Donelan, M. A., 1998: Air-water exchange processes. Physical Processes in Lakes and Oceans, J. Imberger, Ed., Vol. 54, Coastal Estuarine Series, Amer. Geophys. Union, 19-36.

_ 2001: A nonlinear dissipation function due to wave breaking. Proc. Workshop on Ocean Wave Forecasting, Reading, United Kingdom, ECMWF, 87-94.

_ wind-generated waves. Philos. Trans. Roy. Soc. London, 315A, 509-562.

A. V. Babanin, I. R. Young, M. L. Banner, and C. McCormick, 2005: Wave-follower field measurements of the wind-input spectral function. Part I: Measurements and calibrations. J. Atmos. Oceanic Technol., 22, 799-813. 
,,--- , and,- 2006 : Wave-follower measurements of the wind-input spectral function. Part II: Parameterization of the wind input. J. Phys. Oceanogr., 36, 1672-1688.

Drazen, D., W. K. Melville, and L. Lenain, 2008: Inertial scaling of dissipation in unsteady breaking waves. J. Fluid Mech., 611, 307-332.

Duncan, J. H., 1981: An experimental investigation of breaking waves produced by a towed hydrofoil. Proc. Roy. Soc. London, 377A, 331-348.

Evans, K. C., and A. C. Kibblewhite, 1990: An examination of fetch-limited wave growth off the west coast of New Zealand by a comparison with the JONSWAP results. J. Phys. Oceanogr., 20, 1278-1296.

Filipot, J.-F., F. Ardhuin, A. Babanin, and M. Rudi, 2008: Paramétrage du déferlement des vagues dans les modèles spectraux: approches semi-empirique et physique. Proc. National Colloquium on Coastal and Civil Engineering, Sophia Antipolis, France, Centre Francais du Littoral, 335-344.

- — - and —, 2010: A unified deep-to-shallow-water spectral wave-breaking dissipation formulation. Part I: Breaking probability. J. Geophys. Res., in press.

Forristall, G. Z., 1981: Measurements of a saturation range in ocean wave spectra. J. Geophys. Res., 86, 8075-8084.

Garratt, J. R. D., 1977: Review of drag coefficients over oceans and continents. Mon. Wea. Rev., 105, 915-929.

Gemmrich, J. R., 2005: On the occurrence of wave breaking. Rogue Waves: Proc. 'Aha Huliko'a Hawaiian Winter Workshop, Honolulu, HI, University of Hawaii at Manoa, 123-130.

_- M. L. Banner, and C. Garrett, 2008: Spectrally resolved energy dissipation rate and momentum flux of breaking waves. J. Phys. Oceanogr., 38, 1296-1312.

Greenslade, D. J. M., 2001: A wave modelling study of the 1998 Sydney to Hobart yacht race. Aust. Meteor. Mag., 50, 53-63.

Guan, C. L., and L. Xie, 2004: On the linear parameterization of drag coefficient over sea surface. J. Phys. Oceanogr., 34, $2847-$ 2851.

Hasselmann, K., and Coauthors, 1973: Measurements of windwave growth and swell decay during the Joint North Sea Wave Project (JONSWAP). Dtsch. Hydrogr. Z., A8 (Suppl.), $1-95$.

Hwang, P. A., and D. W. Wang, 2004: An empirical investigation of source term balance of small scale surface waves. Geophys. Res. Lett., 31, L15301, doi:10.1029/2004GL020080.

Kahma, K. K., and C. J. Calkoen, 1992: Reconciling discrepancies in the observed growth of wind-generated waves. J. Phys. Oceanogr., 22, 1389-1405.

Komen, G. J., S. Hasslemann, and K. Hasselmann, 1984: On the existence of a fully developed wind-sea spectrum. J. Phys. Oceanogr., 14, 1271-1285.

Liu, P. C., H. S. Chen, D.-J. Doong, C. C. Kao, and Y.-J. G. Hsu, 2007: Monstrous ocean waves during typhoon Krosa. Ann. Geophys., 26, 1327-1329.

Manasseh, R., A. V. Babanin, C. Forbes, K. Rickards, I. Bobevski, and A. Ooi, 2006: Passive acoustic determination of wavebreaking events and their severity across the spectrum. J. Atmos. Oceanic Technol., 23, 599-618.

Melville, W. K., and P. Matusov, 2002: Distribution of breaking waves at the ocean surface. Nature, 417, 58-63.
— F. Feron, and C. J. White, 2002: The velocity field under breaking waves: Coherent structures and turbulence. J. Fluid Mech., 454, 203-233.

Phillips, O. M., 1958: Spectral and statistical properties of the equilibrium range in wind-generated waves. J. Fluid Mech., 156, 505-531.

1966: The Dynamics of the Upper Ocean. Cambridge University Press, $261 \mathrm{pp}$.

_ 1984: On the response of short ocean wave components at a fixed number to ocean current variations. J. Phys. Oceanogr., 14, 1425-1433.

— , F. L. Posner, and J. P. Hansen, 2001: High resolution radar measurements of the speed distribution of breaking events in wind-generated ocean waves: Surface impulse and wave energy dissipation rates. J. Phys. Oceanogr., 31, 450-460.

Pushkarev, A., D. Resio, and V. E. Zakharov, 2003: Weak turbulent approach to the wind-generated gravity sea waves. Physica D, 184, 29-63.

Resio, D. T., C. E. Long, and C. L. Vincent, 2004: Equilibriumrange constant in wind-generated wave spectra. J. Geophys. Res., 109, C01018, doi:10.1029/2003JC001788.

Stiassnie, M., Y. Agnon, and P. A. E. M. Janssen, 2007: Temporal and spatial growth of wind waves. J. Phys. Oceanogr., 37, $106-114$.

Tolman, H. L., and D. Chalikov, 1996: Source terms in a thirdgeneration wind wave model. J. Phys. Oceanogr., 26, 24972518.

Tracy, B. A., and D. T. Resio, 1982: Theory and calculation of the nonlinear energy transfer between sea waves in deep water. U.S. Army Engineer Waterways Experiment Station Rep. N11, $54 \mathrm{pp}$.

Tsagareli, K. N., 2008: Numerical investigation of wind input and spectral dissipation in evolution of wind waves. Ph.D. thesis, University of Adelaide, $217 \mathrm{pp}$.

—, A. V. Babanin, D. J. Walker, and I. R. Young, 2010: Numerical investigation of spectral evolution of wind waves. Part I: Wind-input source function. J. Phys. Oceanogr., 40, 656-666. van der Westhuysen, A. J., M. Zijlema, and J. A. Battjes, 2007: Nonlinear saturation-based whitecapping dissipation in SWAN for deep and shallow water. Coastal Eng., 54, 151-170.

van Vledder, G. P., 2002: Improved parameterisations of nonlinear four wave interactions for applications in operational wave prediction models. Alkyon Rep. 151, 55 pp.

— 2006: The WRT method for computation of non-linear fourwave interactions in discrete spectral wave models. Coastal Eng., 53, 223-242.

Webb, D. J., 1978: Nonlinear transfer between sea waves. Deep-Sea Res., 25, 279-298.

Young, I. R., and L. A. Verhagen, 1996: The growth of fetch limited waves in water of finite depth. Part II: Spectral evolution. Coastal Eng., 28, 79-100.

, and A. V. Babanin, 2006: Spectral distribution of energy dissipation of wind-generated waves due to dominant wave breaking. J. Phys. Oceanogr., 36, 376-394.

_, M. L. Banner, M. A. Donelan, A. V. Babanin, W. K. Melville, F. Veron, and C. McCormick, 2005: An integrated system for the study of wind wave source terms in finite depth water. J. Atmos. Oceanic Technol., 22, 814-828. 